\title{
142.
}

\section{NUMERICAL TABLES SUPPLEMENTARY TO SECOND MEMOIR ON QUANTICS.}

[Now first published (1889).]

IN the present paper I arrange in a more compendious form and continue to a much greater extent the tables (first of each pair) given Nos. 35-39 of my Second Memoir on Quantics, 141, pp. 260-264, which relate to the cubic, the quartic and the quintic functions; and I give the like tables for the sextic, the septimic and the octavic functions respectively. The cubic table exhibits the coefficients of the several $x z$ terms of the function $1 \div\left(1-z .1-x z .1-x^{2} z .1-x^{3} z\right)$, or, what is the same thing, it gives the number of partitions of a given number into a given number of parts, the parts being $0,1,2,3$, (repetitions admissible): or again, regarding the letters $a, b, c, d$, as having the weights $0,1,2,3$ respectively, it shows the number of literal terms of a given degree and given weight. And similarly for the quartic, quintic, sextic, septimic and octavic tables respectively, the parts of course being $0,1, \ldots$ up to 4,5 , 6,7 or 8 , and the letters being $a, b, \ldots$ up to $e, f, g, h$ or $i$. The extent of the tables is as follows:

\begin{tabular}{|c|c|c|c|c|}
\hline $\begin{array}{l}\text { cubic tab } \\
\text { guartic }\end{array}$ & ex & & पर & $\begin{array}{l}18-27 \\
18-36\end{array}$ \\
\hline $\begin{array}{l}\text { tic } \\
\text { tic }\end{array}$ & & & $\begin{array}{l}\text { " } \\
,,\end{array}$ & $18-45$ \\
\hline & & & ” & $15-45$ \\
\hline & " & & " & $12-42$ \\
\hline & & & , & $10-40$ \\
\hline
\end{tabular}

viz. for the quintic, the sextic and the octavic functions these are the deg-weights of the highest invariants respectively. I designate the Tables as the $a d_{-}, a e_{-}, a f_{-}$, ag-, $a h$ - and $a i$-tables respectively.

It is to be noticed that in the several tables the lower part of each column is for shortness omitted; the column has to be completed by taking into it the series 
of bottom terms of each of the preceding columns: thus in the af- or quintic table the complete column for degree 3 would be

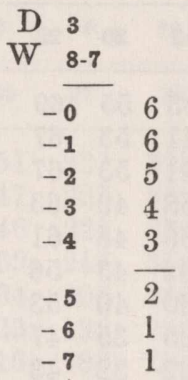

where the concluding terms 2, 1, 1 are the bottom terms of the three preceding columns respectively. And the meaning is that for degree 3 , and weight 8 , or 7 , the number of terms is $=6$; for weight $7-1,=6$, the number of terms is $=6$; and similarly for weights $5,4,3,2,1,0$ the numbers are $5,4,3,2,1,1$; the numbers are those of the terms

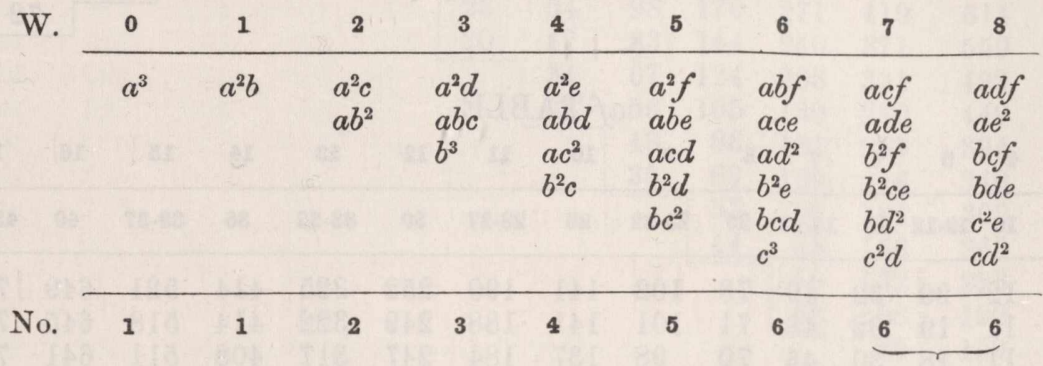

The like remarks and explanations apply to the other tables.

ad-TABLE.

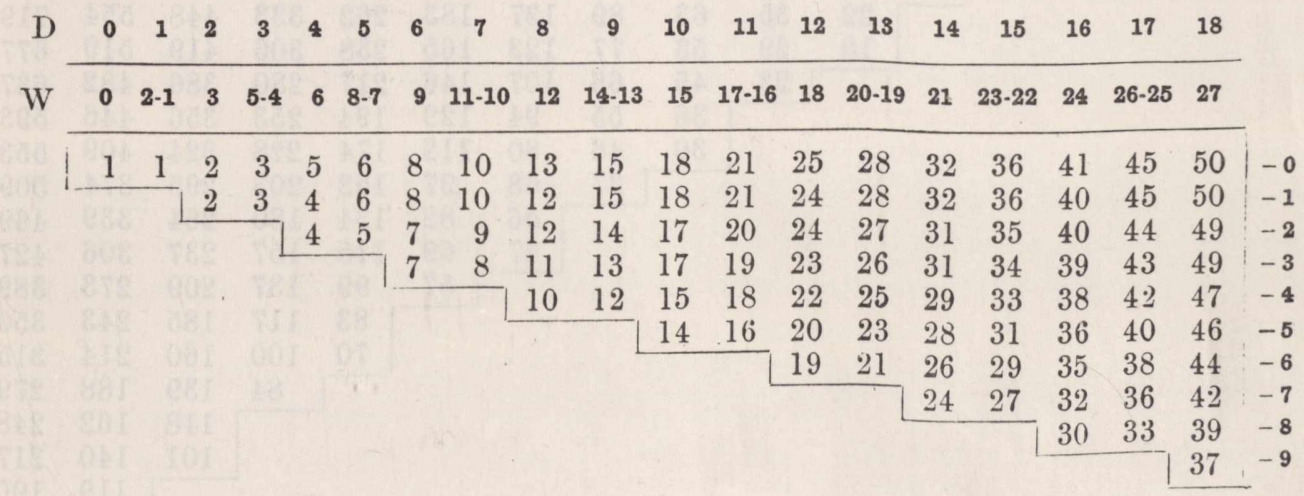


$a e$-TABLE.

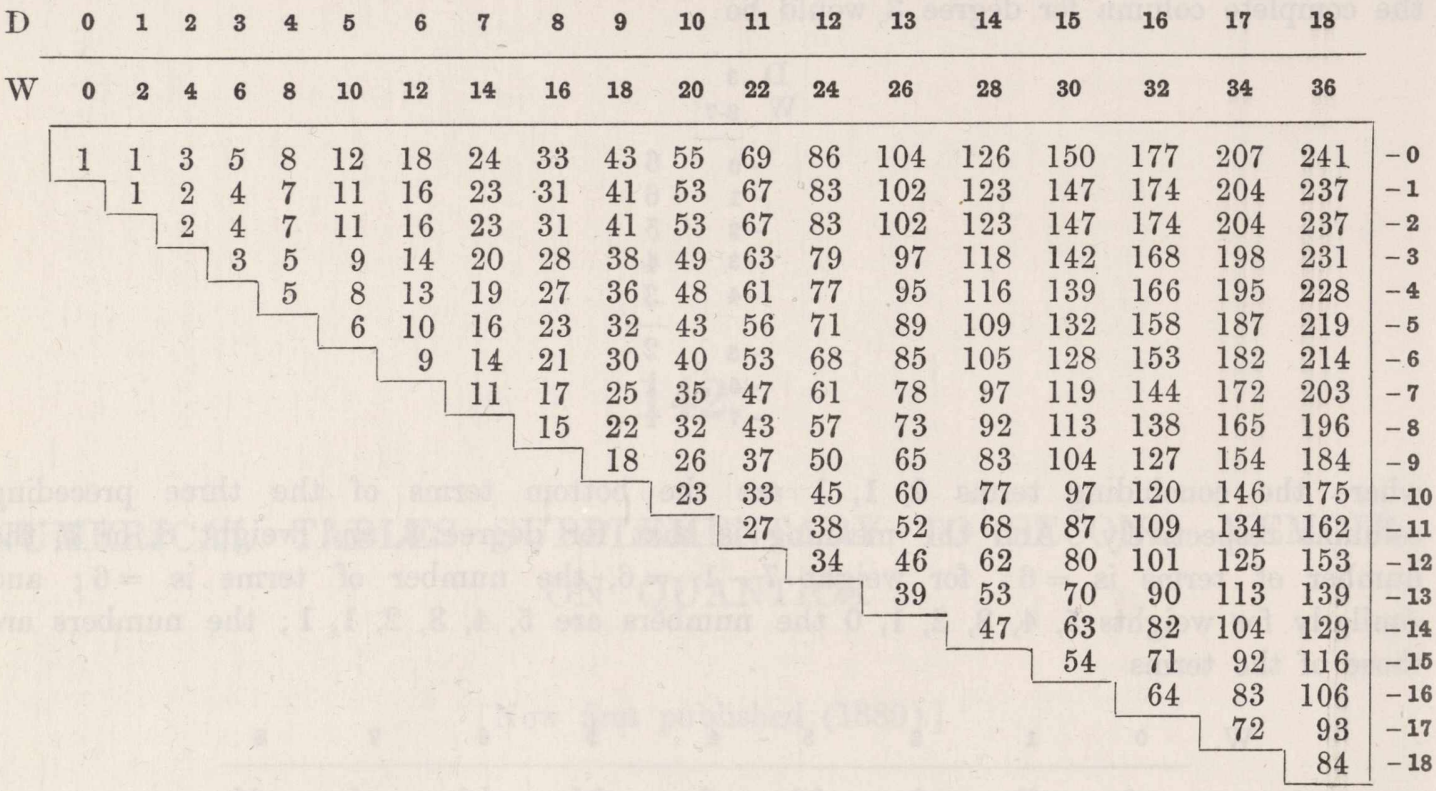

af-TABLE.

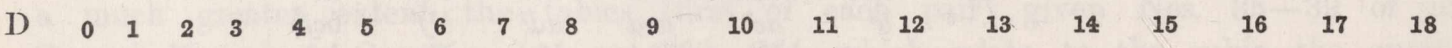

$\begin{array}{llllllllllllllllllll}\mathrm{W} & 0 & 3-2 & 5 & 8-7 & 10 & 13-12 & 15 & 18-17 & 20 & 23-22 & 25 & 28-27 & 30 & 33-32 & 35 & 38-37 & 40 & 43-42 & 45\end{array}$

\begin{tabular}{|c|c|c|c|c|c|c|c|c|c|c|c|c|c|c|c|c|c|c|}
\hline 1 & 1 & 3 & 6 & 12 & 20 & 32 & 49 & 73 & 102 & 141 & 190 & 252 & 325 & 414 & 521 & 649 & 795 & 967 \\
\hline & 1 & 3 & 6 & 11 & 19 & 32 & 48 & 71 & 101 & 141 & 188 & 249 & 322 & 414 & 518 & 645 & 791 & 966 \\
\hline & & 2 & 5 & 11 & 18 & 30 & 46 & 70 & 98 & 137 & 184 & 247 & 317 & 408 & 511 & 641 & 783 & 957 \\
\hline & & 2 & 4 & 9 & 16 & 29 & 43 & 66 & 93 & 134 & 178 & 240 & 309 & 402 & 501 & 630 & 770 & 948 \\
\hline & & & 3 & 8 & 14 & 25 & 39 & 63 & 88 & 127 & 170 & 233 & 299 & 390 & 488 & 619 & 754 & 930 \\
\hline & & & & 6 & 11 & 23 & 35 & 57 & 81 & 121 & 161 & 222 & 286 & 379 & 472 & 601 & 734 & 912 \\
\hline & & & & 5 & 9 & 19 & 30 & 52 & 74 & 111 & 150 & 212 & 272 & 362 & 453 & 583 & 711 & 886 \\
\hline & & & & & 7 & 16 & 26 & 45 & 66 & 103 & 139 & 197 & 256 & 346 & 433 & 559 & 684 & 860 \\
\hline & & & & & & 12 & 21 & 40 & 58 & 92 & 126 & 184 & 239 & 325 & 409 & 536 & 655 & 827 \\
\hline & & & & & & 10 & 17 & 33 & 50 & 83 & 114 & 168 & 220 & 306 & 385 & 507 & 623 & 795 \\
\hline & & & & & & & 13 & 28 & 43 & 72 & 101 & 154 & 202 & 283 & 359 & 480 & 590 & 756 \\
\hline & & & & & & & & 22 & 35 & 63 & 89 & 137 & 183 & 262 & 333 & 448 & 554 & 719 \\
\hline & & & & & & & & 18 & 29 & 53 & 77 & 123 & 165 & 238 & 306 & 419 & 519 & 677 \\
\hline & & & & & & & & & 23 & 45 & 66 & 107 & 146 & 217 & 280 & 386 & 482 & 637 \\
\hline & & & & & & & & & & 36 & 55 & 94 & 129 & 194 & 253 & 356 & 446 & 593 \\
\hline & & & & & & & & & & 30 & 46 & 80 & 112 & 174 & 228 & 324 & 409 & 553 \\
\hline & & & & & & & & & & & 37 & 68 & 97 & 152 & 203 & 295 & 374 & 509 \\
\hline & & & & & & & & & & & & 56 & 82 & 134 & 180 & 264 & 339 & 469 \\
\hline & & & & & & & & & & & & 47 & 69 & 115 & 157 & 237 & 306 & 427 \\
\hline & & & & & & & & & & & & & 57 & 99 & 137 & 209 & 273 & 389 \\
\hline & & & & & & & & & & & & & & 83 & 117 & 185 & 243 & 350 \\
\hline & & & & & & & & & & & & & & 70 & 100 & 160 & 214 & 315 \\
\hline & & & & & & & & & & & & & & & & & & 219 \\
\hline & & & & & & & & & & & & & & & & & 140 & 217 \\
\hline & & & & & & & & & & & & & & & & & 119 & 190 \\
\hline & & & & & & & & & & & & & & & & & & $\begin{array}{l}163 \\
141\end{array}$ \\
\hline
\end{tabular}


$a g$-TABLE.

\begin{tabular}{|c|c|c|c|c|c|c|c|c|c|c|c|c|c|c|c|c|}
\hline 0 & 1 & 2 & 3 & 4 & 5 & 6 & 7 & 8 & 9 & 10 & 11 & 12 & 13 & 14 & 15 & \\
\hline 0 & 3 & 6. & 9 & 12 & 15 & 18 & 21 & 24 & 27 & 30 & 33 & 36 & 39 & 42 & 45 & \\
\hline 1 & 1 & 4 & & 18 & 32 & 58 & 94 & 151 & 227 & 338 & 480 & 676 & 920 & 1242 & 1636 & -0 \\
\hline & 1 & 3 & 8 & 16 & 32 & 55 & 94 & 147 & 227 & 332 & 480 & 668 & 920 & 1232 & 1635 & -1 \\
\hline & 1 & 3 & 7 & 16 & 30 & 55 & 90 & 146 & 221 & 330 & 471 & 664 & 907 & 1226 & 1617 & -2 \\
\hline & 2 & 2 & 7 & 14 & 29 & 51 & 88 & 139 & 217 & 319 & 464 & 648 & 896 & 1203 & 1601 & -3 \\
\hline & 8 & 2 & 5 & 13 & 25 & 48 & 81 & 134 & 205 & 310 & 446 & 634 & 870 & 1182 & 1565 & -4 \\
\hline & 0 & & 4 & 10 & 23 & 42 & 76 & 123 & 196 & 293 & 431 & 608 & 847 & 1145 & 1533 & -5 \\
\hline & $2=$ & & 3 & 9 & 19 & 39 & 68 & 116 & 182 & 280 & 408 & 587 & 813 & 1113 & 1483 & -6 \\
\hline & $y$ & & & 6 & 16 & 32 & 61 & 103 & 169 & 258 & 387 & 553 & 780 & 1064 & 1435 & -7 \\
\hline & a & a & & 5 & 12 & 28 & 52 & 94 & 152 & 241 & 359 & 525 & 737 & 1021 & 1373 & -8 \\
\hline & 10 & 18 & & -1 & 10 & 22 & 46 & 81 & 139 & 218 & 335 & 488 & 699 & 965 & 1316 & -9 \\
\hline & 95 & 7 & & & 7 & 18 & 37 & 71 & 121 & 199 & 304 & 455 & 650 & 914 & 1244 & -10 \\
\hline & $15=$ & 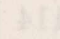 & & & & 13 & 31 & 59 & 107 & 175 & 278 & 415 & 607 & 852 & 1178 & -11 \\
\hline & (65) & & & & & 11 & 24 & 51 & 91 & 157 & 248 & 382 & 557 & 798 & 1102 & -12 \\
\hline & 3 & 02 & & & & & 19 & 40 & 78 & 134 & 222 & 341 & 512 & 733 & 1031 & -13 \\
\hline & $a^{2}$ & 17 & & & & & 14 & 33 & 64 & 117 & 193 & 308 & 462 & 677 & 952 & -14 \\
\hline & nat & & & & & & & 25 & 54 & 98 & 170 & 271 & 419 & 614 & 882 & -15 \\
\hline & $2 a$ & 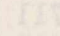 & & & & & & 20 & 42 & 83 & 144 & 240 & 371 & 559 & 803 & -16 \\
\hline & 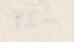 & 82 & & & & & 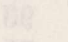 & $x_{2}$ & 34 & 67 & 124 & 206 & 331 & 499 & 734 & -17 \\
\hline & 25 & 320 & & & & & ? & 101 & 26 & 56 & 103 & 180 & 289 & 449 & 661 & -18 \\
\hline & ef & & & & & & & & & 43 & 86 & 151 & 253 & 394 & 596 & -19 \\
\hline & (9) & te & & & & & 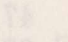 & & & 35 & 69 & 129 & 216 & 349 & 529 & -20 \\
\hline & race & & & & & & & & & & 57 & 106 & 187 & 302 & 472 & -21 \\
\hline & $x^{3}$ & & & & & & & & & & 44 & 88 & 156 & 263 & 412 & -22 \\
\hline & 8 & 695 & & & & & & & & & & 70 & 132 & 223 & 362 & -23 \\
\hline & $x^{2}$ & & & & & & & & & & & 58 & 108 & 192 & 303 & -24 \\
\hline & 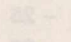 & & & & & & & & & & & & 89 & 159 & 270 & -25 \\
\hline & $a^{2}$ & & & & & & & & & & & & 71 & 134 & 228 & -26 \\
\hline & 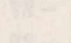 & & & & & & & & & & & & & 109 & 195 & -27 \\
\hline & 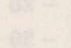 & & & & & & & & & & & & & 90 & 161 & -28 \\
\hline & 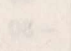 & & & & & & & & & & & & & & $\begin{array}{l}135 \\
110\end{array}$ & $\begin{array}{l}-29 \\
-30\end{array}$ \\
\hline
\end{tabular}


$a h$-TABLE.

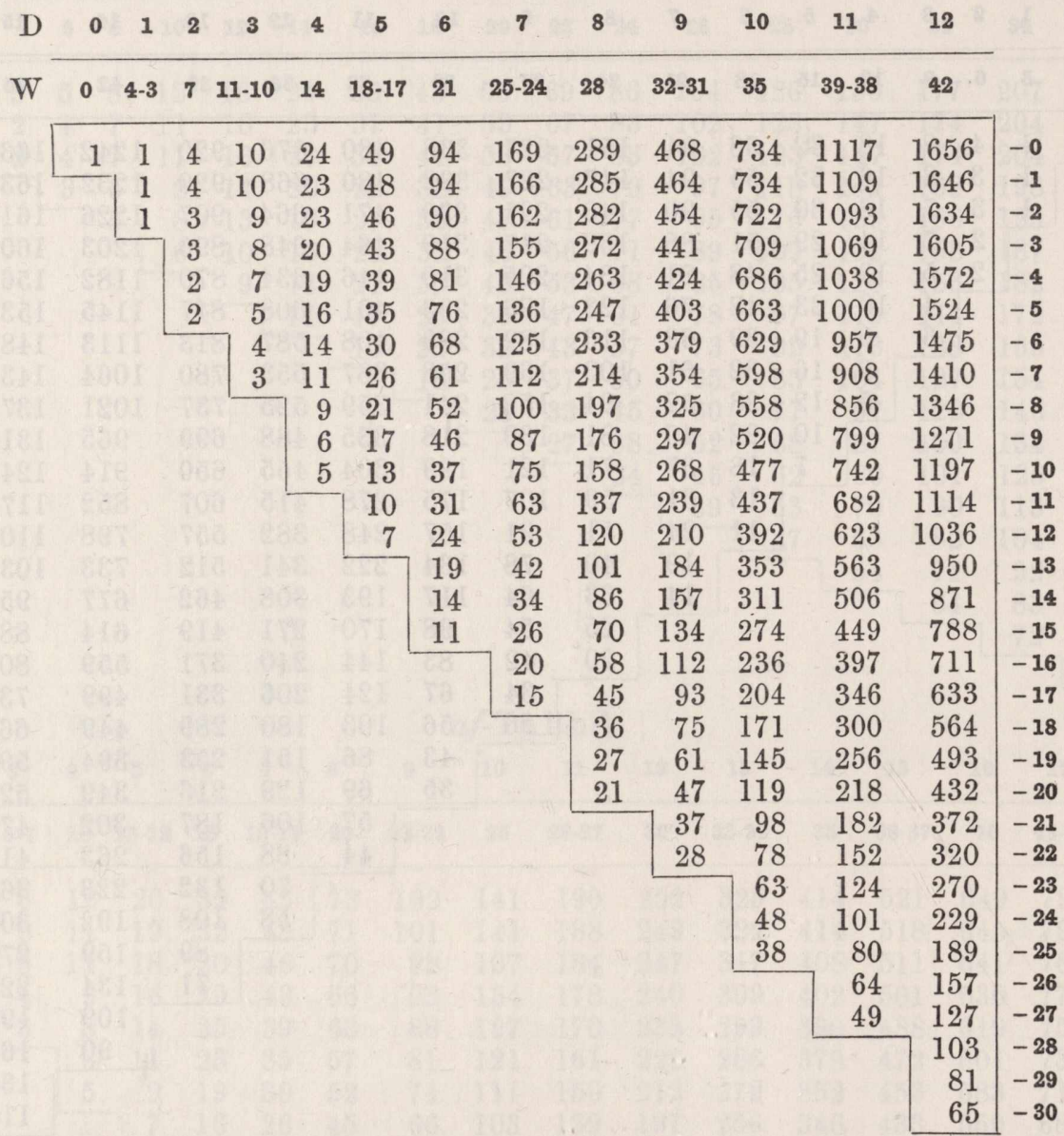


ai-TABLE.

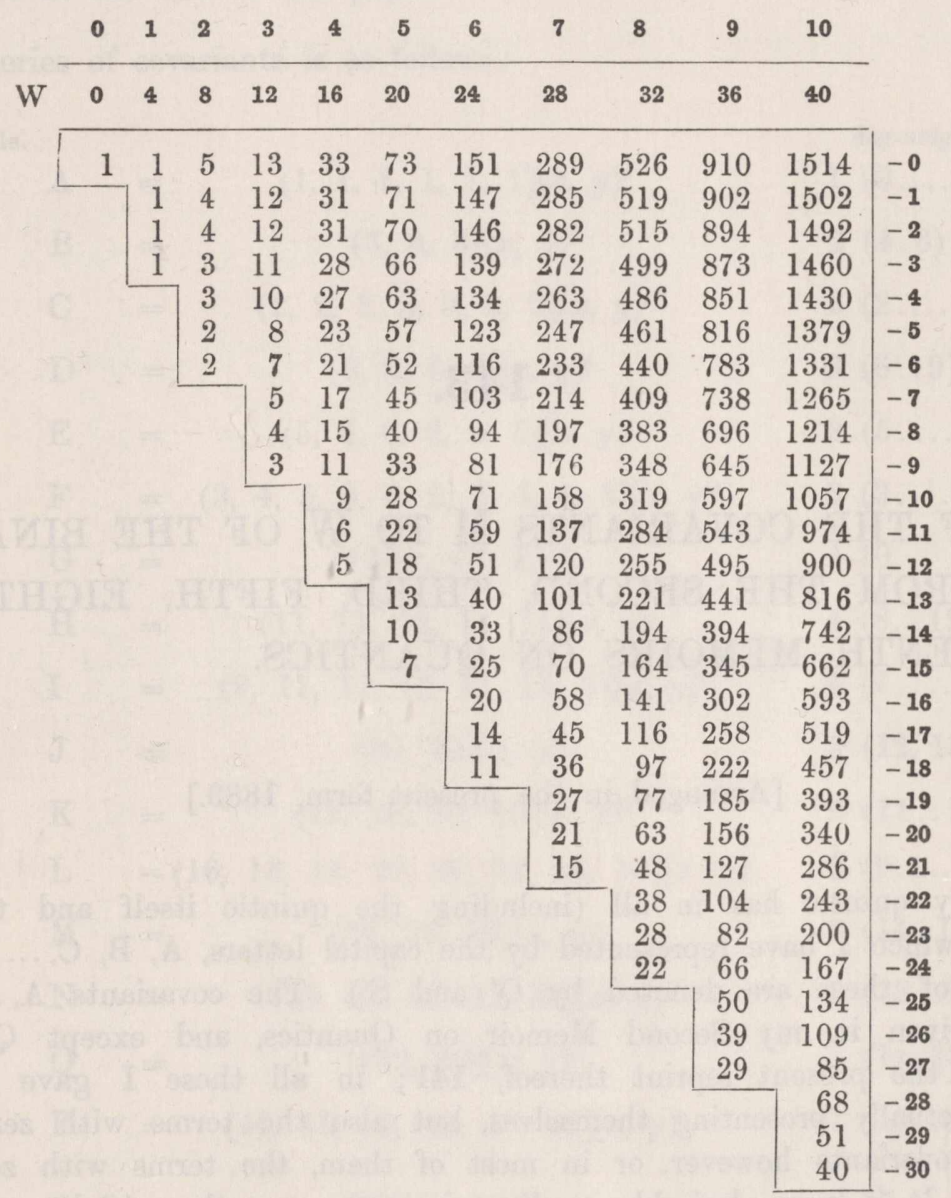

The numbers of each table are connected in several ways with those of the preceding tables. One of these connexions, which is of some importance, is best explained by an example: in the af-table, 8-20, the number of terms of degree 8 and weight 20 is 73 ; and we have $73=1+6+16+23+27$, viz. (see p. 288) these are the numbers of the terms in $a^{4}, a^{3}, a^{2}, a^{1}, a^{0}$ respectively: the complementary factors, (for example) of $a^{3}$ are $b e f^{3}$, \&c. terms in $b, c, d, e, f$ of the degree 5 and weight 20, and (replacing therein each letter by that which immediately precedes it) these are in number equal to the terms in $a, b, c, d, e$ of the degree 5 and weight $20-5,=15$; thus the number 6 of the terms in question is that for the deg-weight 5-15 of the ae-table: and so $1,6,16,23,27$ are the numbers in the ae-table for the deg-weights 4-16, 5-15, 6-14, 7-13 and 8-12 respectively, or (making a change rendered necessary by the abbreviated form of the tables) say for the deg-weights 4-0, 5-10, 6-14, 7-13 and 8-12.

C. II. 